\title{
Network Environment Design of Computer Personality Psychology Based on the Personalized Data Mining Method
}

\author{
Zhibing Zhong ${ }^{1}$, Jurong Yang ${ }^{2}$, Xiaofei Xiao ${ }^{3, *}$ \\ ${ }^{1}$ Psychological Couseling Center, Jiangxi University of Traditional Chinese Medicine Nanchang, \\ 330004, China \\ ${ }^{2}$ School of Humanity, Jiangxi University of Traditional Chinese Medicine Nanchang, 330004, China \\ ${ }^{3}$ Higher Education Research Office, Jiangxi University of Traditional Chinese Medicine Nanchang, \\ 330004, China
}

Keywords: Data Mining; System Evaluation; Personality Psychology; Network

\begin{abstract}
Data Mining Technology is defined as a method to extract the original, unknown and potentially useful information and knowledge hiding in the data collection. Through data mining method, system analysis method, experimental method and other methods, this paper conducts the research on the test and evaluation network of personality psychology adaptability scale and develops computer evaluation system of personality psychology adaptability. By experimental verification, the computer evaluation system of personality psychology adaptability possesses features of security, operability, stability, accuracy and reasonable hierarchy management.
\end{abstract}

\section{Introduction}

Through test on mass personality psychology adaptability scale, evaluation on the basis of feedback information is a significant link in the research of personality psychology adaptability. Currently, in the test and evaluation implementation process of personality psychology adaptability scale, with small controlling difficulty and less collective information, data collection still uses question-answering method by paper and needs to be done under the guidance of psychological counseling experts; the statistical process is conducted before completely classification and assignment artificially, which leads to lower speed and more mistakes in the operating process and determines the option scores and factor scores; from perspective of the preservation of collective information, collection by paper is likely to be worn and lost. All above-mentioned factors may lead to the lower credibility and validity in the research conducted by psychological counseling experts and scientific research personnel[1-3]. For instance, traditional test and evaluation network of personality psychology adaptability scale is to give out answer sheets to the mass, instruct them to fill out the basic information, leads them to read test contents and help them answer the questions by psychological counseling experts. Upon completion, psychological counseling experts extract question options of answer sheets according to the various factors, assign and calculate total scores by summation on the basis of factor classification. Then, the validity of the scale test is determined in the light of total scores of the scale credibility [4]. If the test is valid, the corresponding determining period of factor scores should be found and the evaluation analysis of personality psychology adaptability should be conducted finally. In the test and evaluation process, the evaluation is far from objectivity and accuracy because the several evaluations of the same tester or the same evaluation of various testers were conducted by different experts with subjectivity. At the same time, the integrity of test data cannot be guaranteed due to the various time intervals. The application of personality psychology adaptability scale makes it easier for experts to regulate the personality state and do their best. However, the paper test by manpower cannot timely make feedback of the personality state. It proposes requirements for us to solve problems of the speed, accuracy of the controlling and fractional statistics and the intuition of the feedback information.

With the rapid development of computer science and network technology, relying on their own accuracy, quickness, intelligence and other features, information technology and data mining technology have quickly integrated into other research areas and have become the indispensable 
tools for researchers. And the rise of the computer network for the psychological evaluation of the mass psychology of computer adaptive assessment provided [5-7]. The main subject on the basis of standardized scale mass psychological adaptation, based on the testing and evaluation process scale transformation into a computer model of the standard operation, the manual conversion statistics are now widely used by the computer automatically statistics, and provide to the tester and measured by the visual evaluation of information.

\section{Multi-process Simulation of Data Mining}

Data mining refers to the process of extracting advanced information and knowledge which are new and unknown before with potential use from among large amounts of incomplete, noisy, fuzzy and random data. The knowledge can be represented in the forms of concept, rule, law and mode.

(1) Multi-stage Process Model on the Basis of Data Mining

Data mining is a multi-step processing procedure [8]. A typical data mining system usually includes data preparation (sampling selection, pre-processing and data conversion), data mining as well as result interpretation and evaluation, as is shown in the following Figure 1.

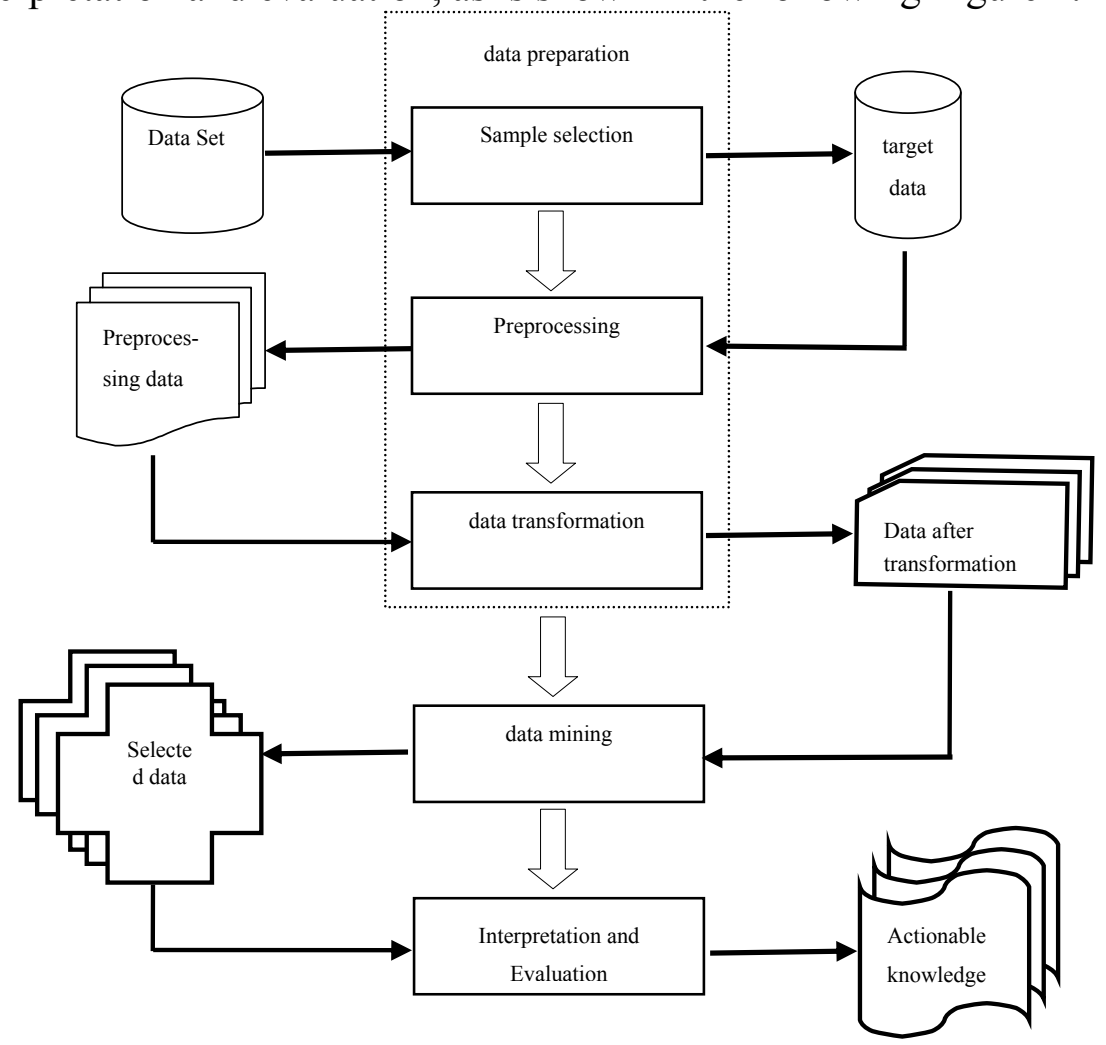

Figure 1: Multi-stage Process Model of Data Mining

Due to complexity, repeatability and incompleteness of original data, it is essential to follow four principles in data preparation: subject-oriented, processing data to be meaningful, avoidance of bad effect and principle of efficiency. As to data preparation, it mainly includes data sampling selection, data pre-processing, data reduction, data fusion and data conversion. Operations on data such as partitioning, normalization, induction, rotation and projection are essential to meet requirements by specific mining tools or algorithm so as to enhance mining efficiency. Data partitioning: partitioning of chunk data is beneficial to index and monitoring as well as to reorganization and reconstruction. Data normalization: in normalized method of attribute maximum-minimum value, supposing is the maximum attribute value and is the minimum attribute value, the benefit attribute is shown by formula (1) as follows:

$$
v_{i}^{\prime}=\left\{\begin{array}{ll}
\frac{\max \left(v_{i}\right)-v_{i}}{\max \left(v_{i}\right)-\min \left(v_{i}\right)} & \text { if } \max \left(v_{i}\right)-\min \left(v_{i}\right) \neq 0 \\
1 & \text { if } \max \left(v_{i}\right)-\min \left(v_{i}\right)=0
\end{array}\right\}
$$

Normalization is conducted to cost-type attribute by adopting formula 2. 


$$
v_{i}^{\prime}=\left\{\begin{array}{lr}
\frac{v_{i}-\operatorname{mix}\left(v_{i}\right)}{\max \left(v_{i}\right)-\min \left(v_{i}\right)} & \text { if } \max \left(v_{i}\right)-\min \left(v_{i}\right) \neq 0 \\
1 & \text { if } \max \left(v_{i}\right)-\min \left(v_{i}\right)=0
\end{array}\right\}
$$

(2) Data Mining

In the stage of data mining algorithm implementation, the mining tasks or purposes including classification, cluster, association rules discovery or sequence pattern discovery should be determined first according to definitions of problems. After determining mining tasks, relevant algorithm to be used should be determined. There are two factors that should be considered in selection of algorithm. First, suitable mining algorithm should be selected as different data have different characteristics. Second, algorithm should be selected according to requirements by users or actual operation system. For instance, some users expect for descriptive and easy-to-understand knowledge, while some others require for predictive knowledge of the highest possible prediction accuracy with little attention paid to understandability of knowledge acquired.

Construction of Computer Evaluation System for Popular Psychological Adaptability Scale

Requirement Analysis of Functions of Computer Evaluation System for Popular Psychological Adaptability Scale

1) Function of Psychological Counseling Experts Management

After logging in the system, psychological counseling experts can conduct management over registration information of testing users and inquire all testing results and reports of each testing user. The psychological counseling experts can input testing data of previous answer sheet by hand, and can modify basic information relevant to the individual person.

2) Testing Function of Testing Users

The testing users can obtain testing permission by registration, and can select question type (full-questionnaire test or individual factor test) in test selection area after logging in the system and reading test notice. After that, the test can be finished by selecting options and an automatic testing report will be produced by the system upon submitting answers. The report can be printed with testing data and report saved in background database. The testing players can modify his/her own registration information.

3) Function of Report Printing

Testing users and psychological counseling experts can print testing results report (the report will include player's basic information, testing date, testing option score, factor classification score, psychological stage and evaluation analysis of each factor as well as norm linear contrast chart).

4) Function of Historical Information Query

Through the system platform, the psychological counseling experts can inquire all result information (that is, the result reports in all evaluations) in different periods concerning each testing user.

(2) The implement of computer measurement system for the adaptability scale of public mental

1) The implement of the table structure of system database

Through analyzing the process of testing and evaluating the adaptability scale of public mental, it can determine the table structure of system database of the public mental adaptability scale computer measurement system. The database consists of 11 tables, such as the information table of system administrator, scale factors classification management table, the information management table of psychological consultants, the management table of evaluating answer, and so on. All test data and basic setup information of system will be stored in the relevant data tables. Between one table and another table, it will set up a relevant relation depended on main index value and external key value.

For example 'test scale information management table' includes eight fields, such as ID, tsid, question, Answer A, Answer B, Answer C, Answer D, and Flag (in table 1). Through the table, it should manage the examination question and evaluating answer of the scale table. 
Table 1 test scale information management

\begin{tabular}{|c|c|c|c|c|}
\hline $\begin{array}{c}\text { The name of } \\
\text { chart }\end{array}$ & Function & $\begin{array}{l}\text { Containing } \\
\text { field }\end{array}$ & Data type & Description \\
\hline \multirow{8}{*}{ question list } & \multirow{8}{*}{$\begin{array}{c}\text { Test scale } \\
\text { information }\end{array}$} & ID & Automatic Number & \\
\hline & & tsid & Number & $\begin{array}{c}\text { Factor subject to the } \\
\text { question }\end{array}$ \\
\hline & & question & Text & Test content \\
\hline & & Answer A & Text & Scores when choosing A \\
\hline & & Answer B & Text & Scores when choosing B \\
\hline & & Answer C & Text & Scores when choosing $\mathrm{C}$ \\
\hline & & Answer D & Text & Scores when choosing D \\
\hline & & Flag & Number & The question is valid or not \\
\hline
\end{tabular}

2) The implementation of evaluation system

For example, to test the user engaged in sports. If the test user selected the test content, select system should choose the test data in the database, and display test content.

If the test user submitted the answer, the system should automatically evaluate the test options, and classify statistics. According to the score of each factor to find relevant score section in normal model, and determine the phase factor and evaluation of relevant psychological. It will show the test result in report form (if the credibility score of the test users was below 30 points, the system should remind, and do not show the result of statistical analysis for the test user).

\section{The test results and analysis on the stability of the verification of measurement system}

To choose 25 students from one university, at the same time they should install, register, login, manage, modify, and test the measurement system on different computers, in order to validate the functions of system module and fluency of system. The results of statistical verification were shown in table 2:

Table 2 to verify stability of system

\begin{tabular}{|c|c|c|}
\hline test project & Normal rate $(\%)$ & Abnormal rare $(\%)$ \\
\hline Installation and operation & 100 & 0 \\
\hline register and login & 100 & 0 \\
\hline Management setting for managers & 100 & 0 \\
\hline $\begin{array}{c}\text { Management on psychological counseling } \\
\text { experts }\end{array}$ & 100 & 0 \\
\hline Selection and retrieval of test scale & 100 & 0 \\
\hline Assignment statistics of the answers & 100 & 0 \\
\hline Print display of scale reporting & 100 & 0 \\
\hline Historical data input & 100 & 0 \\
\hline Classification search & 100 & 0 \\
\hline
\end{tabular}




\begin{tabular}{|c|c|c|}
\hline The retrieval of test scale & 100 & 0 \\
\hline The comparison chart of factor normal model & 100 & 0 \\
\hline
\end{tabular}

The test user can operate system smoothly; each function of measurement system fully meets the design requirements of system function before researching; at the same time it makes the assessment process of paper mental adaptability scale fully shown in the computer measurement system.

\section{Conclusion}

In this paper, the research is based on the functions of computer measurement system public of psychological adaptability scale on data mining, for example collecting user's information and management, selecting and testing index, automatically evaluating points and statistical evaluation, displaying normal contrast curve, displaying report forms and printing them, and testing the information query, and other functions. The paper has a good practical significance. The article shows an effective method, and will greatly improve the scope of mind evaluation in China.

\section{Bibliography}

[1] Xiyu Ji. The application examples of data mining technology [M]. Beijing: China Machine Press 2009.

[2] Shuqing Ma. The algorithm research of association rules in data mining [D]. Jilin University, 2007.

[3] Chen An, Chen Ning, Longxiang Zhou. The technology and application of data mining [M]. Beijing: science press, 2006.

[4] Jiang Lei. Cluster analysis application in the books management system [J]. Journal of intelligence research, 2010 (10): 111-113.

[5] Ruliang Zhang. The improved and application in clustering analysis of algorithm mining [D]. Guizhou University, 2004.

[6] Wenwei Chen. The course of data warehouse and data mining [M]. Beijing: Tsinghua University Press, 2006.

[7] Yunping Shi. K - Means algorithm analysis ofusing the average error criterion function E [J]. Computer and information technology, 2008 (6): 21-23.

[8] Xinsheng Wang, Yufei Gu. The public psychological training and control [M]. Beijing: Beijing sport university press, 2004.01 